\title{
A Comparative Study of Different Template Matching Techniques for Twin Iris Recognition
}

\author{
Shahreen Kasim", Rohayanti Hassan ${ }^{*}$, Nor Sukriyah Mohd ${ }^{*}$, Rohaizan Ramlan ${ }^{+}$, Hairulnizam Mahdin", \\ Mohd Farhan Md Fudzee ${ }^{\#}$ \\ ${ }^{\#}$ Soft Computing and Data Mining Centre, Faculty of Computer Science and Information Technology, \\ Universiti Tun Hussein Onn, Johor, Malaysia
}

\begin{abstract}
"Software Engineering Research Group, Faculty of Computing, Universiti Teknologi Malaysia, 81310 Skudai, Johor, Malaysia E-mail: rohayanti@utm.my
\end{abstract}

${ }^{+}$Department of Production and Operation Management, Universiti Tun Hussein Onn Malaysia 86400 Parit Raja, Batu Pahat, Johor, Malaysia

\begin{abstract}
Biometric recognition is gaining attention as most of the organization is seeking for a more secure verification method for user access and other security application. There are a lot of biometric systems that exist which are iris, hand geometry, and fingerprint recognition. In the biometric system, iris recognition is marked as one of the most reliable and accurate biometric in term of identification. However, the performance of iris recognition is still doubted whether the iris recognition can generate higher accuracy when involving twin iris data. So, specific research by using twin data only needs to be done to measure the performance of recognition. Besides that, a comparative study is carried out using two template matching technique which are Hamming Distance and Euclidean Distance to measure the dissimilarity between the two iris template. From the comparison of the technique, better template matching technique also can be determined. The experimental results showed that iris recognition could distinguish twin as it can distinguish two different, unrelated people as a result obtained showed the good separation between intra and interclass and both techniques managed to obtain high accuracy. From the comparison of template matching technique, Hamming Distance is chosen as a better technique with low False Rejection Rate, low False Acceptance Rate and high Total Success Rate with the value of $\mathbf{2 . 5 \%}, \mathbf{8 . 7 5 \%}$, and $96.48 \%$ respectively.
\end{abstract}

Keywords — iris recognition; twin; Hamming distance; Euclidean distance

\section{INTRODUCTION}

Nowadays, the system recognized people by their PIN code, password, and signatures. But, in this modern digital era, it is very easy to forget the identification by hacking their PIN or password if the security of the system is not secure enough. So, the biometric system provides the solution to this problem. Among varies of the biometric system exist, iris recognition is considered as the most promising technique that can provide higher accuracy identification [1]. This is because of the iris is rich with unique pattern and texture that will not change significantly during the individual lifetime and contain many significant features that can act as a quantifiable parameter for the measurement in the identification process. Iris comes from the Greek word which means the representation of the rainbow. It is a thin circular diaphragm, which located behind the cornea and in front of the lens. The irises contain many tiny tissues that represent in furrows, crypts, corona, collagenous fibres, serpentine vasculature, freckles, striations, pits, and rift.

Twin has the same allele which makes them genetically identical. There are two types of twins, which are dizygotic (non-identical) and monozygotic (identical). Hollingsworth et al. [2] have concluded from the experiment that there are several similarities in twin iris texture. The necessity for iris recognition systems to precisely decide the identity of individual who has a twin is increasing as the number of twins is increased by the years.

Template matching technique is carried out after the image processing [3] and feature extraction [4]. It is the crucial stage where the template image is matched with the image from the database to identify the person. There are two types of comparison which are intraclass and interclass. Intraclass is the process of comparing different template of the same person while interclass is the process of comparing different person. The result of both comparisons should give 
distinct values to yields high accuracy in iris recognition. In this study, there is two template matching technique being applied which are Hamming Distance and Euclidean Distance. The performances of both techniques are being compared to determine better template matching technique and also were used as the measurement to assess the accuracy in twin iris recognition.

Hamming distance gives a fractional measure of disagreeing bits between two equal lengths of binary patterns. The main operator in the HD equation is Exclusive OR (XOR) gate. The XOR will produce true output (1) when only one of the compared input bits is true. If both of the input is false (0) or true (1), the result yields to false (0) output. Euclidean Distance is derived from the old Pythagoras theorem and is defined as the linear distance between two points in the dimensional space. The result of Euclidean Distance is the shortest distance between the two binary strings of the template.

Hollingsworth et al. [2] have concluded from the experiment that there are similarities in twin iris texture. Daugman [5] stated that the twin irises are different, but Hollingsworth et al. [2] argued that there is no specific research has been carried out to compare the twin irises in some sense to effectively discover the accuracy of recognition. Wildes [6] has generated higher accuracy when using 60 iris data from 40 subjects including several twins image, but it is not clear how many from those data are twins, to sum up to the conclusion that there is no error when matching twin iris image [6]. So, the problem arises whether the iris recognition can generate higher accuracy during the identification of twin. So, the specific research needs to be done focusing only on twin iris image data to find out the accuracy. Besides that, the template matching technique also is being compared to find a better technique that will produce higher accuracy when involving twin iris data.

This study has motivated to measure the accuracy of template matching technique and to assess overall performance in identification of twin in iris recognition by analyze the effect of different template matching technique for twin in iris recognition and designing the suitable template matching techniques which are Hamming Distance and Euclidean Distance to obtain the accuracy for twin iris recognition.

Template matching technique evaluates the template that is generated from the feature extraction process. It is used to measure the dissimilarity value for the comparison [7] and aimed to minimize the false match of different person and maximize the probability of true person for identification. This process has two modes which are one-to-one matching and one-to-many matching [1].

Hamming Distance (HD) is a number used to signify the distinction between two binary strings. It permits the computer to distinguish and correct the error on its own. Hamming Distance has the capability to detect up to two bits in error and correct single bit error. In iris recognition, HD is mainly used in the template matching technique. The popularity of HD had increased since Daugman [5] has proposed the technique in his iris recognition invention and the result produced was very promising. Since that, most of the researcher implemented HD in their biometric research.
The main function of HD in iris recognition is to compare two-bit pattern generated from feature extraction technique. The result or distance value produced from this technique will act as the measurement metric to determine whether the irises come from the same or different person [8]. The main operation in the HD is the exclusive OR (XOR) and AND operator. XOR operator will evaluate the binary string and AND operator will only choose the bits that are not corrupted by the noise such as eyelids, eyelashes, and reflection [9]. XOR operator yields a true result if exactly one of two conditions is true. Fereira [9] stated that the minimum HD value would be chosen as the final matching result. Each iris region produces a bit pattern from another iris since an individual iris region contains freedom with a high degree of features. Furthermore, two iris codes that come from the same iris are highly correlated.

There are several researchers had addressed the advantages of HD performance in iris recognition system. According to [10], HD is a fast and simple technique. It is also very versatile since it has been tested in numerous image data types such as twin image, noisy image, and noncooperative image. Besides that, Khan [11] stated that HD shows excellent processing and handling speed compared to another technique. The required times for HD to produce is less since it has a simple algorithm. Gupta [12] stated that HD is more reasonable technique compared to others. There is only one disadvantage pointed out by the previous researcher, i.e. it has a problem when dealing with applying codes for large blocks.

HD is widely used in iris recognition research. Ma [13] had used HD to evaluate the accuracy of their proposed method that regards iris texture as a signal and adapted the wavelet transform technique to process those signals. The HD result showed good performance in processing the data. Daugman [14] had implemented HD to evaluate his new method which is Fourier based technique that is used to solve the trigonometry problem in iris. Matey [15] had proposed new method to reduce the constraint of iris position in the image segmentation stage, and he used the HD to match the template of iris. The result of HD showed high accuracy with less of error. Hollingsworth [16] had proposed new technique in template matching which is the hybrid of fragile bit distance with HD and compares the accuracy to the HD technique alone. The performance of that technique is implemented by using twin iris data. Hence, the hybrid of HD and fragile bit distance produced a more promising result. Tisse [17] implemented HD for the verification process to estimate bit to bit agreement to the proposed method that is used to extract the significant feature from the texture of iris. Park and Lee [18] had executed HD to measure the distance of the two iris codes produced from the new extraction method using wavelet transform technique. Barwick [19] performed HD technique to summing the XOR operator to figure out the percentage of incompatible bits when altering the optical transfer function.

Another technique commonly used in the template matching technique is Euclidean Distance (ED). [1] stated that $\mathrm{ED}$ is the most common technique. In most cases, people will refer to ED when dealing with distance. ED can have from one up to n-dimensional spaces. Xu and Xia [20] stated that there is many types and advanced formulation of 
ED. The examples are Weighted Euclidean Distance, Normalized Euclidean Distance, and Modified Average Euclidean Distance. ED is the linear distance between the two points in the metric space. The equation in this technique is referred to the Pythagoras theorem. ED calculates the similarity value of the two iris template generated from feature extraction process [21].

There are two researchers had addressed the advantages of ED performance in iris recognition system. Wang [22] stated that ED is very compatible to be embedded with the classification technique to produce higher accuracy. According to Moi [23], ED is considered as the simple technique since the equation of this technique is only the repeated Pythagoras theorem formula. The disadvantage of this technique is the computational involved in measuring distance is very complex [22]. Khan [11], stated that ED requires a lot of calculation process and requires massive computation process.

Euclidean distance is the most common technique used in the template matching phase [1]. Khan [11] implemented ED to calculate the distance between two feature that is extracted with new proposed method 1D Gabor Filter. Patil [24] used ED to measure the iris pattern similarity when comparing the performance of three feature extraction technique. To measure the newly proposed method, Manisha and Sanjay [25] implemented ED to measure the disagreed of bits when comparing the sample template with database template. Kulkarni [21] had compared the performance of ED with the Libor Masek Algorithm and concluded that Libor Masek had produced higher accuracy. This is because they consider the non-significant value that will raise the number of bit patterns. Shancez [1] had tested the performance of ED by measure the feature that is distinct in value to compare the accuracy of multiscale - zero representation and Gabor Filter technique. Masih [26] executed ED to compute distance in the new feature of representation to classify iris. ED also had been used as the verification technique for the regional approach in iris recognition that only chooses the selected area of iris [27]. Pillai [28] implemented ED to measure the point in the two iris template to evaluate the proposed unified framework based on sparse representation and random projection. The advantages and disadvantages of template matching techniques are summarized in Table 1.

The remainder of this paper is organised as follows. In Section II, the material and methods used are discussed in detail. Section III presents the analysis and the discussion of the experimental results. Finally, Section IV summarises the future work and conclusion.
TABLE I

ADVANTAGES AND DisAdVANTAGES OF TEMPlATE MATCHING TECHNIQUES

\begin{tabular}{|c|c|c|}
\hline Techniques & Advantages & Disadvantages \\
\hline $\begin{array}{l}\text { Hamming } \\
\text { Distance } \\
\text { [10], } \\
{[11],[12],} \\
{[29]}\end{array}$ & $\begin{array}{l}\text { - Fast \& simple } \\
\text { - Versatile } \\
\text { - Excellent } \\
\text { processing and } \\
\text { handling speed } \\
\text { - More reasonable }\end{array}$ & $\begin{array}{l}\text { Has problem } \\
\text { when dealing with } \\
\text { applying coders } \\
\text { for large blocks }\end{array}$ \\
\hline $\begin{array}{l}\text { Euclidean } \\
\text { Distance } \\
{[22],[23],} \\
{[11]}\end{array}$ & $\begin{array}{l}\text { - Great compatibility } \\
\text { - Simple }\end{array}$ & $\begin{array}{l}\text { Require massive } \\
\text { computation } \\
\text { process }\end{array}$ \\
\hline
\end{tabular}

\section{MATERIAL AND METHOD}

The data for this research is 320 iris images for both left and right position of 20 pairs of twin (40 individual). Each individual has 8 iris images that are picked randomly from 3183 total images from CASIA-TWIN database. In this research, the intraclass comparison is performed first before interclass for both left and right eyes position. For intraclass, two different templates of the same individual are used as input while for interclass, two different templates of that individual with his/her corresponding twin are used as input. Before the iris sample is matching with the database, the iris sample must first be processed that involved three step. The first step is image segmentation by using Hough Transform to isolate the noise and locate the circular iris region. Next, is iris normalization by using Daugman Rubber Sheet Model to produce iris with the same dimension to get the exact iris region. The last step in image pre-processing is feature extraction by using Gabor Filter to extract the significant feature in the iris and change those features into bits string. After that, the binary string is being matched in template matching stage. Hamming Distance and Euclidean Distance is applied to calculate the distance or dissimilarity between the two binary strings. The lowest distance is picked as the final value for each intra and interclass comparison. The binary string is shifted to both left and right position to get the lowest distance. The result of both techniques is illustrated in the line graph. Lastly, the accuracy of both techniques is evaluated by using FAR, FRR and TSR. The better technique is determined by the technique that obtains lower FAR, lower FRR and higher TSR. The overall system is illustrated in Fig. 1. 


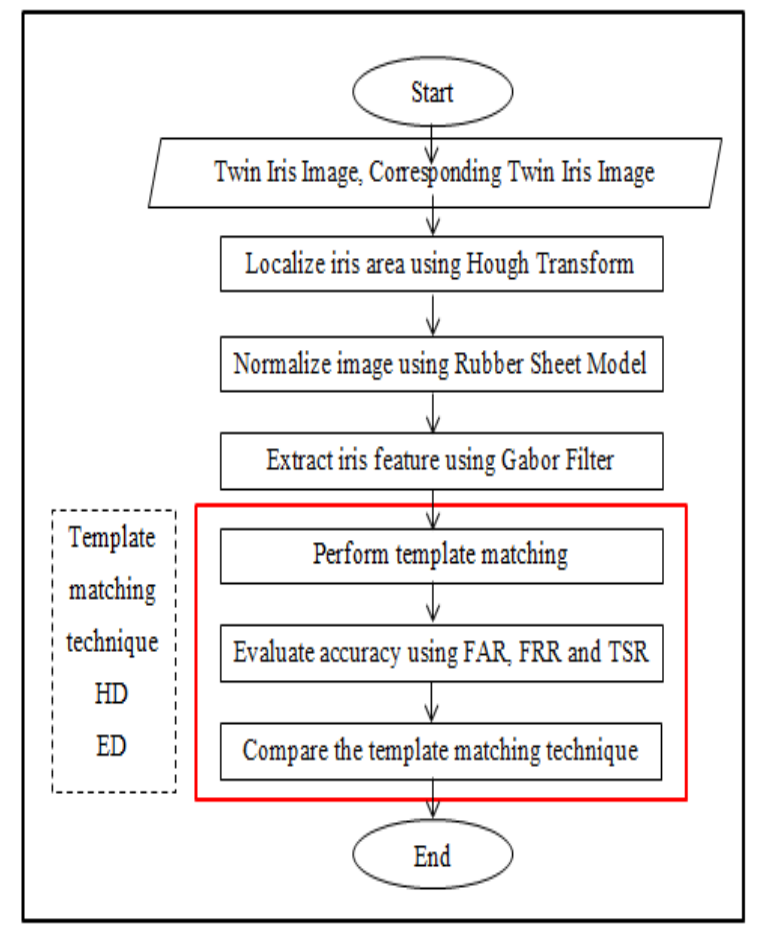

Fig. 1 Flowchart for twin iris recognition

\section{A. Hamming Distance}

Hamming distance (HD) gives a fractional measure of disagreeing bits between two binary patterns. The two binary patterns are derived from the template of the sample, and another one comes from the template of the database that is generated during feature extraction stage. HD only can produce distance when the two-bit patterns are equal. The main operator in the HD equation is Exclusive OR (XOR) gate which will determine the disagree value when comparing the two input bits. The XOR will produce true output (1) when only one of the compared input bits is true. If both of the input is false (0) or true (1), the result yields to false (0) output. Table 2 below shows the summarization of XOR logic gate.

TABLE II

XOR LOGIC GATE

\begin{tabular}{|c|c|c|}
\hline \multicolumn{2}{|c|}{ Input } & Output \\
\hline A & B & A XOR B \\
\hline 0 & 0 & 0 \\
\hline 0 & 1 & 1 \\
\hline 1 & 0 & 1 \\
\hline 1 & 1 & 0 \\
\hline
\end{tabular}

Let $\mathrm{A}$ and $\mathrm{B}$ be the bits pattern in the template that is being compared. Generally, HD is a calculation of total different bits over the total number of bits in the bits pattern. The equation of HD is defined below:

$$
H D=\frac{1}{N} \sum_{j=1}^{N} A_{j}(\oplus) B_{j}
$$

Where $N$ represents the total number of bits in the bit pattern $A_{j}$ and $B_{j}$ represent the two bits that are being compared, and $\mathbb{Q}$ icon indicates the XOR operator. Table 3 below shows the example of HD operation.

TABLE III

EXAMPLE OF HAMMING DISTANCE

\begin{tabular}{|c|c|c|c|c|c|c|c|c|c|c|c|c|}
\hline $\boldsymbol{A}_{\boldsymbol{j}}$ & $\mathbf{1}$ & $\mathbf{1}$ & $\mathbf{1}$ & $\mathbf{0}$ & $\mathbf{1}$ & $\mathbf{0}$ & $\mathbf{1}$ & $\mathbf{1}$ & $\mathbf{1}$ & $\mathbf{0}$ & $\mathbf{0}$ & $\mathbf{0}$ \\
\hline $\boldsymbol{B}_{\boldsymbol{j}}$ & $\mathbf{1}$ & $\mathbf{1}$ & $\mathbf{1}$ & $\mathbf{0}$ & $\mathbf{0}$ & $\mathbf{1}$ & $\mathbf{0}$ & $\mathbf{1}$ & $\mathbf{0}$ & $\mathbf{0}$ & $\mathbf{1}$ & $\mathbf{1}$ \\
\hline $\boldsymbol{A}_{\boldsymbol{j}}$ XOR $\boldsymbol{B}_{j}$ & 0 & 0 & 0 & 0 & 1 & 1 & 1 & 0 & 1 & 0 & 1 & 1 \\
\hline $\begin{array}{c}\text { Total disagreeing } \\
\text { bits }\end{array}$ & \multicolumn{10}{|c|}{$0+0+0+0+1+1+1+0+1+0+1+1=6$} \\
\hline HD & \multicolumn{10}{|c|}{$\mathbf{1 2}=\mathbf{5}$} \\
\hline
\end{tabular}

Satish [8], stated that the value produced from the calculation of HD would act as the measurement metric to determine whether the irises come from the same or different person. If the two-bit pattern comes from a different person (completely independent), the HD value will be equal to 0.5 . This is because the independence of the two bits will be completely random. There is a chance of 50 percent to set any bit to 1 and 50 percent chance to set any bit to 0 which means half of the bits agree to the compared pattern and another half disagrees. If the two-bit pattern comes from the same person (dependent), the result produced will be close to 0 . 0 . This is because there are highly correlated to each other and the bits would agree between two iris patterns.

In iris recognition, according to [5], the decision criterion or threshold value (confidence) is 0 . 32. If HD value is less or equal to 0.32 , it indicates that the iris comes from the same person while if the HD values is more than 0.32 , it indicates that the iris comes from the different person or left and right eye of the same person.

\section{B. Euclidean Distance}

Euclidean Distance (ED) is widely used when dealing with distance problem. It is defined as the linear between two points in the metric space. It can have from one and up to $\mathrm{n}$-dimensional space. The equation of this technique is derived from the old Pythagoras theorem. It is also referred as the shortest distance between two points as shown below in Fig. 2.

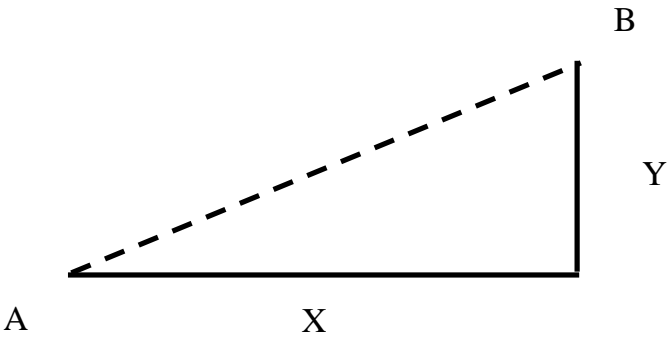

Fig. 2 Euclidean distance theory

To calculate the distance (hypotenuse) between point $A$ and $B$, the equation would be as follow:

$$
d(A, B)=\sqrt{X^{2}+Y^{2}}
$$

Where $X$ represent the differences between points for one feature and $Y$ represent the differences between points for another feature. Generally, if we have two points $(A$ and $B$ ) 
whose feature value are $(a 1, a 2, \ldots$, ai) and $(b 1, b 2, \ldots$, bi), the summarization of equation would be:

$$
d(A, B)=\sqrt{\sum_{i=1}^{n}\left(a_{i}-b_{i}\right)^{2}}
$$

Where $d(A, B)$ represents the Euclidean distance, $\mathrm{n}$ represent the dimension of the feature vector, $\mathrm{a}_{\mathrm{i}}$ represent the $i$ th component of the sample in the feature vector for point $A$ and $b_{i}$ is the $i$ th of the sample in feature vector for point $B$. In conclusion, ED specifies the square root of the sum of squares of differences of every point in separate dimension [30].

In iris recognition, Kulkarni [21], stated that ED would calculate the dissimilarity value of the two iris template generated from feature extraction process. If the two iris template comes from the same individual, the value will be fall in the range lower than the threshold, but in ED their result will never be equal to 0 even the iris comes from the same person or same side of the eye.

\section{RESULT AND DISCUSSION}

Below are the result of Hamming and Euclidean Distance for both intraclass and interclass, as well as for both left and right position illustrated in the line graph and summary for FAR, FRR and TSR.

The line graph in Fig. 3 shows the Hamming distance value for the left eye of the first individual in a pair of twin. Overall, there is a good separation between the intra and interclass. For intraclass, there is no false rejection. This indicates that the iris recognition system when using HD as matching technique accept all the authorized user when comparing left eye of the same individual. While for interclass, there is one false acceptance which is for twin 10 . The HD value when comparing the individual with his/ her corresponding twin is 0.3111. It wrongly accepts the individual as the corresponding twin of that individual.

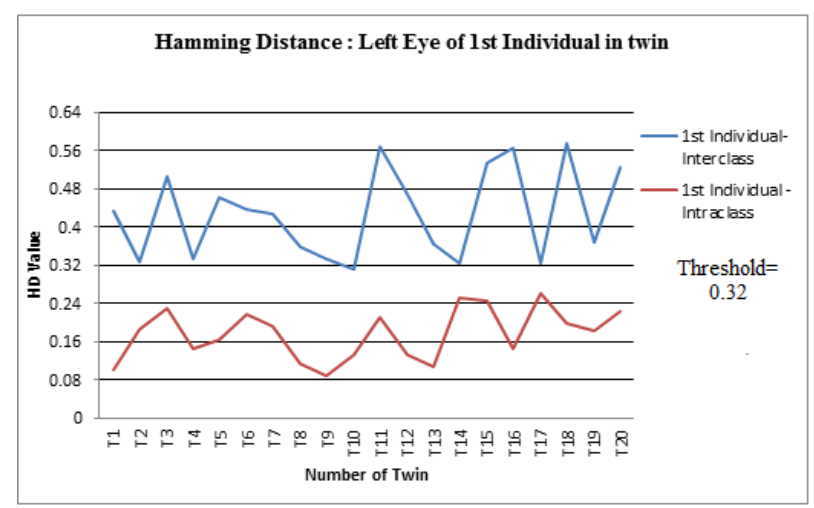

Fig. 3 Hamming distance value for left eyes of the first individual in twin

For the second individual, a different template of the iris was served as a template for the matching process. The line graph in Fig. 4 shows the Hamming distance value for the left eye of the second individual in a pair of twin. Overall, there is good a separation between the intra and interclass. For intraclass, there is still no false rejection. All of the value obtained fall below the threshold. This means that the iris recognition system when using HD as matching technique accept all the authorized user when comparing left eye of the same individual. While for interclass, there are two false acceptances which are for twin 4 and 10. The HD value when comparing the individual with his/ her corresponding twin is 0.3014 and 0.3044 . The iris recognition system wrongly accepts two individual as the corresponding twin of that individual.

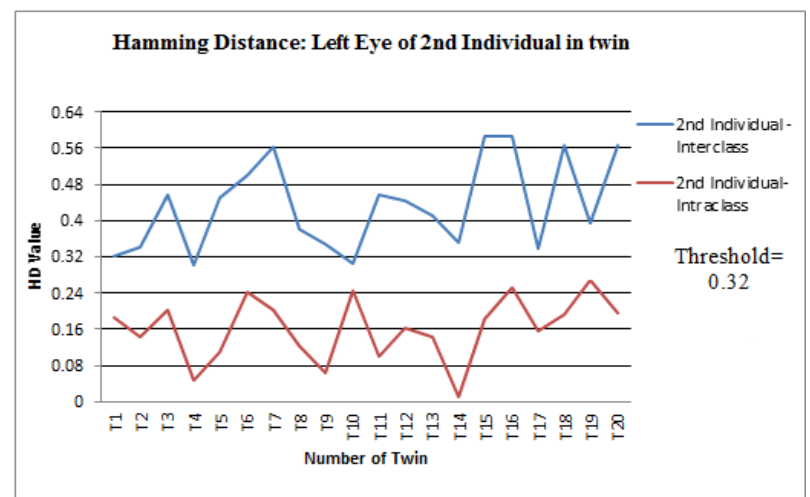

Fig. 4 Hamming distance value for left eyes of the second individual in twin

The line graph in Fig. 5 shows the Hamming distance value for the right eye of the first individual in a pair of twin. Overall, there is a separation between the intra and interclass. For intraclass, there is no false rejection. The iris recognition system accepts all the authorized user when comparing left eye of the same individual. While for interclass, there is one false acceptance which is for twin 11 . The HD value when comparing the individual with his/ her corresponding twin is 0.3042 . The iris recognition system wrongly accepts the individual as the corresponding twin of that individual.

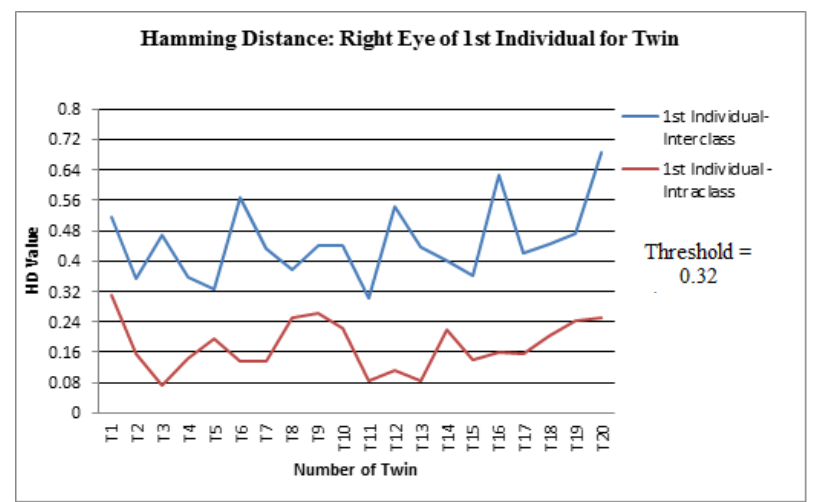

Fig. 5 Hamming distance value for right eyes of the first individual in twin

The line graph in Fig. 6 shows the Hamming distance value for the right eye of the second individual in a pair of twin. Overall, there is a good separation between the intra and interclass. For intraclass, there are two false rejections. This means that the iris recognition system when using HD as matching technique reject two authorized user when comparing right eye of the same individual which are for twin 9 and 19. The HD values when comparing the same individual by using different templates are 0.3415 and 0.3562 . While for interclass, there are two false acceptances which are for twin 5 and 11. The HD value when comparing the individual with his/ her corresponding twin is 0.3192 and 0.3148 . This indicates that the iris recognition system 
wrongly accepts three individual as the corresponding twin of that individual.

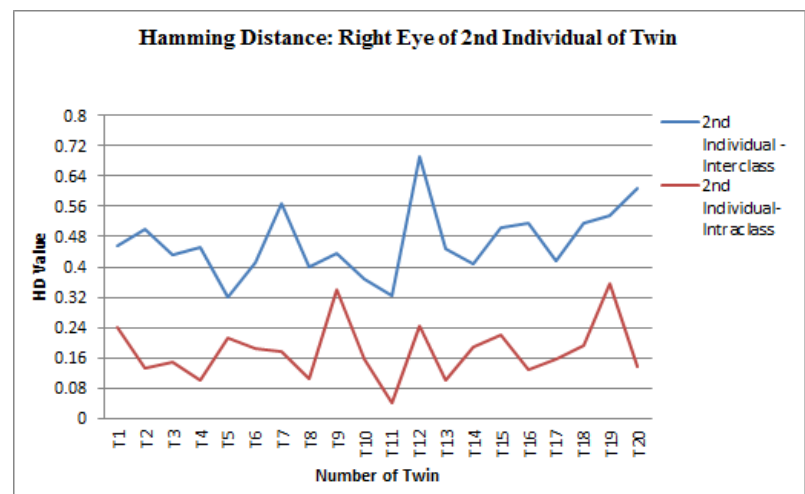

Fig. 6 Hamming distance value for right eyes of the second individual in twin

The line graph in Fig. 7 shows the Euclidean distance value for the left eye of the first individual in a pair of a twin. Overall, there is a separation between the intra and interclass. For intraclass, there is no false rejections. The iris recognition system when using ED as matching techniques accepts all authorized user when comparing left eye of the same individual. The ED value when comparing the same individual by using different template are 3.751 and 4.378 . While for interclass, there are three false acceptances which are for twin 10, 13 and 17. The ED value when comparing the individual with his/her corresponding twin are 3.673, 3.437 and 3.665 respectively. The iris recognition system wrongly accepts three individual as the corresponding twin of that individual.

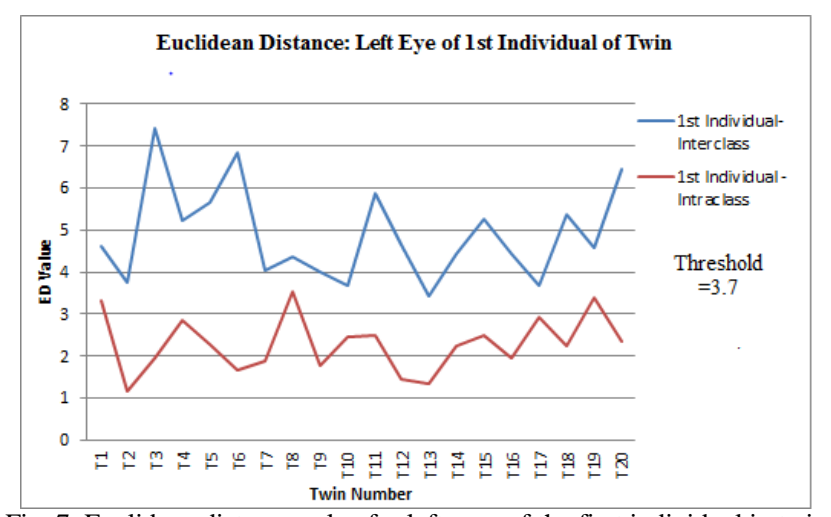

Fig. 7 Euclidean distance value for left eyes of the first individual in twin

The line graph in Fig. 8 shows the Euclidean distance value for the left eye of the second individual in a pair of twin. Overall, there is a separation between the intra and interclass. For intraclass, there are two false rejections. This indicates that the iris recognition system when using ED as matching technique reject two authorized user when comparing left eye of the same individual which is for twin 1 and 8 . The ED value when comparing the same individual by using a different template is 3.714 and 3.761 . While for interclass, there are two false acceptances which are for twin 11 and 13. The ED value when comparing the individual with his/ her corresponding twin are 3.425 and 3.142 respectively. This means that the iris recognition system when using ED as matching technique, wrongly accepts two individual as the corresponding twin of that individual.

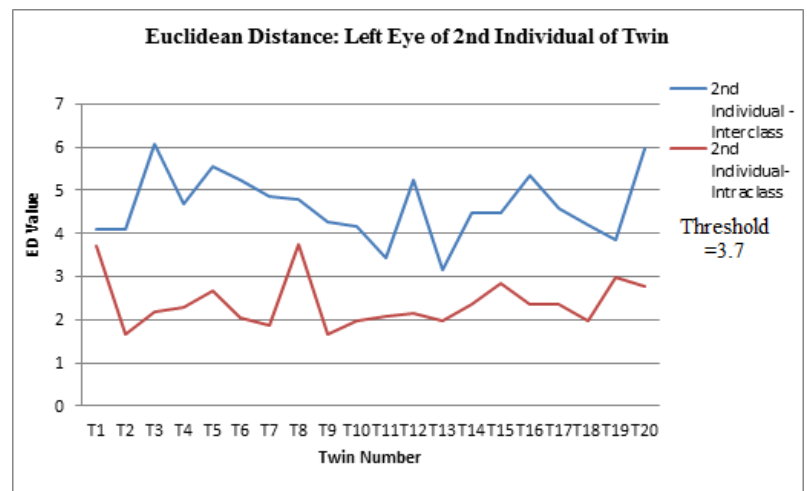

Fig. 8 Euclidean distance value for left eyes of the second individual in twin

The line graph in Fig. 9 shows the Euclidean distance value for the right eye of the first individual in a pair of twin. Overall, there is a separation between the intra and interclass. For intraclass, there is one false rejection. The iris recognition rejects one authorized user when comparing left eye of the same individual which are for twin 17 with 3.758 ED value 3.758. While for interclass, there are three false acceptances which are for twin 1, 5 and 17. The ED value when comparing the individual with his/ her corresponding twin are $3.241,3.578$ and 3.563 respectively. The iris recognition system when using $\mathrm{ED}$ as matching technique wrongly accepts three individual as the corresponding twin of that individual.

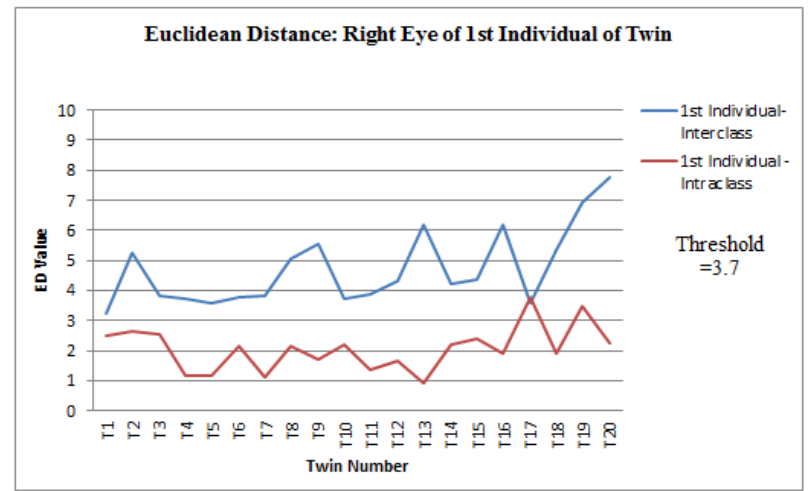

Fig. 9 Euclidean distance value for right eyes of the first individual in twin

The line graph in Fig. 10 shows the Euclidean distance value for the right eye of the second individual in a pair of twin. Overall, there is a separation between the intra and interclass. For intraclass, there are two false rejections. It rejects two authorized user when comparing right eye of the same individual which is for twin 9 and 17. The ED value when comparing the same individual by using a different template is 3.778 and 3.883. While for interclass, there are two false acceptances which are for twin 4 and 17. The ED value when comparing the individual with his/ her corresponding twin are 3.667 and 3.649. The iris recognition system when using ED as matching technique wrongly accepts two individual as the corresponding twin of that individual. 


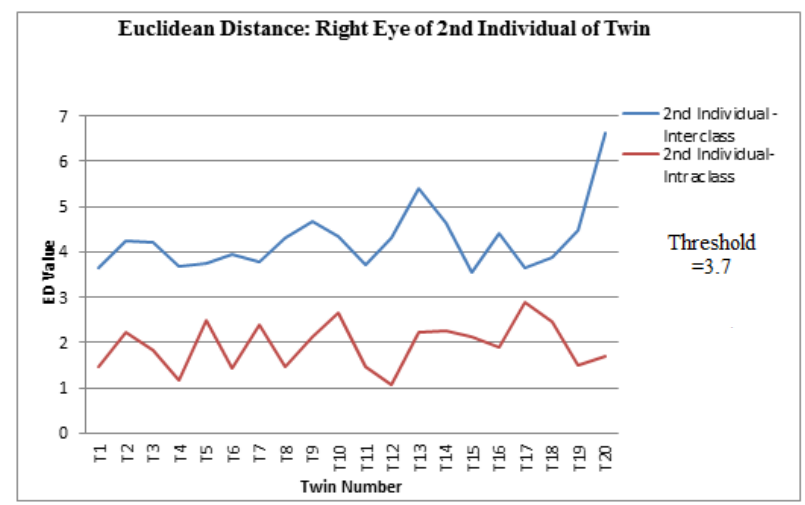

Fig. 10 Euclidean distance value for right eyes of the second individual in twin

For the comparison of techniques, the best technique result is expected to have low FRR, low FAR and high TSR. Low FRR means there is less rejection error when comparing the same person. From the result obtained, the value of FRR for Hamming Distance is $2.5 \%$ while Euclidean Distance is $8.75 \%$. Low FAR means there is less acceptance error when comparing different person/ twin. From the Table 4, Hamming Distance obtained $8.75 \%$ FAR while Euclidean Distance obtained $12.5 \%$ FAR. Lastly, TSR means the overall accuracy of the technique. This value is obtained from the result of FRR and FAR earlier. The TSR for Hamming Distance is $96.48 \%$ while Euclidean Distance is $93.35 \%$. From the value, it is clearly shown that the Hamming Distance performs better as a matching technique than Euclidean Distance with lower FRR, lower FAR and higher TSR which are $2.5 \%, 8.75 \%$, and $96.48 \%$ respectively. Hence, it is proven that it was the most accurate, simple and popular technique used for matching in iris recognition consistent with the previous literature that implements this technique more than another technique exists such as Euclidean Distance, Manhattan Distance, and Mahalanobis Distance.

TABLE IV

SUMMARY OF FRR, FAR AND TSR FOR HAMMING AND EUCLIDEAN DISTANCE

\begin{tabular}{|c|c|c|}
\hline Matching Technique & $\begin{array}{c}\text { Hamming } \\
\text { Distance }\end{array}$ & $\begin{array}{c}\text { Euclidean } \\
\text { Distance }\end{array}$ \\
\hline $\begin{array}{c}\text { Number of False } \\
\text { Rejection }\end{array}$ & 2 & 7 \\
\hline $\begin{array}{c}\text { Number of False } \\
\text { Acceptance }\end{array}$ & 7 & 10 \\
\hline FRR $(\%)$ & 2.5 & 8.75 \\
\hline FAR $(\%)$ & 8.75 & 12.5 \\
\hline TSR (\%) & 96.48 & 93.35 \\
\hline
\end{tabular}

The overall performance of twin iris recognition can be assessed by using two criteria which are the accuracy of both template matching technique and the distribution of intraclass and interclass value. For both left and right eye position, it can be observed that the result is very good. From the calculation of TSR, both techniques manage to obtain higher accuracy which are $96.48 \%$ and $93.36 \%$. This accuracy is quite similar to the result of the previous researcher that obtained the result around $90-100 \%$. Besides that, the distribution of intraclass and interclass also are well separated in every graph although there are several errors in matching. For Hamming Distance, most of the intraclass values fall below 0.32 with can conclude that to iris system, twin iris codes or bit strings that are produced during feature extraction during image pre-processing are as different as two different individual iris codes. This indicates that the iris recognition system can distinguish the twin to some extent as it can distinguish two different individuals who does not have any twin. Two matching errors and most of the interclass values fall above 0.32 with seven matching error. While for Euclidean Distance, most of the intraclass values fall below 3.7 with 7 matching errors and most of the interclass value fall above 3.7 with 10 matching errors.

\section{CONCLUSIONS}

The twin iris image from CASIA database was used as a dataset to carry out the experimental result. The performance measurement of FAR, FRR and TSR have proven that Hamming Distance is more accurate technique than Euclidean Distance. It gave lower FAR and FRR which were $8.75 \%$ and $2.5 \%$ respectively. This technique also obtained higher TSR which is $96.48 \%$ than Euclidean Distance. So, it was proven that this technique is reliable and accurate. From the performance of both techniques which obtain high accuracy and well-separated distribution of intraclass and interclass value, it can be concluded that iris recognition can distinguish a pair of twin very well as a result shown very well - separation between intraclass and interclass. For future work, image enhancement technique such as Clip Limited Adaptive can be applied in Histogram Equalization in order to fix the contrast for the eye image. From the database aspect, there is still lack of database that provides twin iris images. Only CASIA database is the open source storage that provides the image to the public. So, the comparison cannot be made with another database to obtain more reliable and accurate result. The effort to create and share the database to the public especially for identical and non-identical twin image needs to be increased. In future development, this research could be enhanced by referring to various other works available such as [31]-[39].

\section{ACKNOWLEDGMENT}

This research was funded by Research Contact Grant, Vot No: 4C907. Also many thanks to collaborative sponsor, GATES Scholars Foundation of GATES IT Solution Sdn. Bhd. and to RMC, UTM.

\section{REFERENCES}

[1] C. Sanchez-avila and R. Sanchez-reillo, "Two different approaches for iris recognition using Gabor filters and multiscale zero-crossing representation," Pattern Recognit., vol. 38, pp. 231-240, 2005.

[2] K. Hollingsworth, K. W. Bowyer, and P. J. Flynn, "Similarity of iris texture between identical twins," in 2010 IEEE Computer Society Conference on Computer Vision and Pattern Recognition Workshops, CVPRW 2010, 2010, pp. 22-29.

[3] R. Nilakant, H. P. Menon, K Vikram, "A Survey on Advanced Segmentation Techniques for Brain MRI Image Segmentation," International Journal on Advanced Science, Engineering and Information Technology, vol 7, no. 4, 2017.

[4] Yuhandri, S. Madenda, E. P. Wibowo, Karmilasari, "Object Feature Extraction of Songket Image Using Chain Code Algorithm," International Journal on Advanced Science, Engineering and Information Technology, vol 7, no. 1, pp. 235-241, 2017. 
[5] J. Daugman, "How iris recognition works," Circuits Syst. Video Technol. IEEE Trans., vol. 14, no. 1, pp. 21-30, 2004.

[6] R. R. P. Wildes, J. J. C. Asmuth, G. L. G. Green, S. C. Hsu, R. J. Kolczynski, J. R. Matey, and S. E. McBride, "A system for automated iris recognition," Proc. 1994 IEEE Work. Appl. Comput. Vis., pp. 121-128, 1994.

[7] S. S. Mabrukar, N. S. Sonawane, J. A. Bagban, and I. I. I. Preprocessing, "Biometric System using Iris Pattern Recognition," Int. J., no. 5, pp. 54-57, 2013.

[8] G. Sathish, "Multi-algorithmic IRIS Recognition," Int. J. Comput. Appl., vol. 38, no. 11, pp. 13-21, 2012.

[9] A. Ferreira, A. Lourenco, B. Pinto, and J. Tendeiro, "Modifications and Improvements on Iris Recognition," Biosignals 2009 Proc. Int. Conf. Bio-Inspired Syst. Signal Process., pp. 72-79, 2009.

[10] S. Patil, S. Gudasalamani, and N. Iyer, "A survey on Iris recognition system," Electr. Electron., 2016.

[11] M. T. Khan, D. Arora, and S. Shukla, "Feature extraction through iris images using 1-D Gabor filter on different iris datasets," in 2013 6th International Conference on Contemporary Computing, IC3 2013, 2013, pp. 445-450.

[12] S. Gupta, V. Doshi, Jain Abhinav, and Iyer Sreeram, "Iris Recognition System using Biometric Template Matching Technology," Int. J. Comput. Appl., vol. 1, no. 2, pp. 24-28, 2010.

[13] T. T. Li Ma, Y. W., and D. Z., "Efficient iris recognition by Charactering Key Local Variation,” IEEE Trans. Image Process., vol. 13, no. 6, pp. 739-750, 2004.

[14] J. Daugman, "New Methods in Iris Recognition," IEEE Trans. Syst. Man, Cybern. Part B Cybern., vol. 37, no. 5, pp. 1167-1175, 2007.

[15] J. R. Matey, O. Naroditsky, K. Hanna, R. A. Y. Kolczynski, D. J. Loiacono, S. Mangru, M. Tinker, T. M. Zappia, and W. Y. Zhao, "Iris on the move: Acquisition of images for iris recognition in less constrained environments," Proc. IEEE, vol. 94, no. 11, pp. 19361946, 2006

[16] K. Hollingsworth, K. W. Bowyer, S. Lagree, S. P. Fenker, and P. J. Flynn, "Genetically identical irises have texture similarity that is not detected by iris biometrics," Comput. Vis. Image Underst., vol. 115, no. 11 , pp. 1493-1502, 2011.

[17] C. Tisse, L. Martin, L. Torres, M. Robert, and others, "Person identification technique using human iris recognition," in Proc. Vision Interface, 2002, pp. 294-299.

[18] J. Park and C. Lee, "Robust Iris Recognition with Region Division," Image (Rochester, N.Y.), vol. 5672, pp. 161-168, 2005.

[19] D. S. Barwick, "Increasing the information acquisition volume in iris recognition systems.," Appl. Opt., vol. 47, no. 26, pp. 4684-91, 2008.

[20] Z. Xu, Z. Xu, and M. Xia, "Distance and similarity measures for hesitant fuzzy sets," Inf. Sci. (Ny)., vol. 181, no. 11, 2011.

[21] S. B. Kulkarni, R. S. Hegadi, and U. P. Kulkarni, "Improvement to libor masek algorithm of template matching method for iris recognition," Proc. Int. Conf. Work. Emerg. Trends Technol. ICWET' 11, no. January 2011, p. 1270, 2011.

[22] L. Wang, Y. Zhang, and J. Feng, "On the Euclidean Distances of Images," IEEE Trans. Pattern Anal. Mach. Intell., vol. 27, no. 8, pp.1334-1339, 2005.

[23] S. H. Moi, N. B. A. Rahim, P. Saad, P. L. Sim, Z. Zakaria, and S. Ibrahim, "Iris biometric cryptography for identity document," in SoCPaR 2009 - Soft Computing and Pattern Recognition, 2009, pp. 736-741.

[24] P. S. Patil, S. R. Kolhe, and R. V. Patil, "The Comparison of Iris Recognition using Principal Component Analysis, Log Gabor and Gabor Wavelets," Int. J. Comput. Appl., vol. 43, no. 1, pp. 29-33, 2012.
[25] M. Khaladkar and S. Ganorkar, "A Novel Approach for Iris Recognition,” Int. J. Adv. Res. Comput. Eng. Technol., vol. 1, no. 4, pp. 2278-1323, 2012.

[26] K. Masih, C. Chandrakar, and M. Sharma, "International Journal Of Engineering Sciences \&Amp; Management Iris Recognition System Using Stastical Feature Extraction,” Int. J. Engg. Sci. Mgmt.(, 2012.

[27] D. Impedovo, G. Pirlo, and L. Scianatico, "Regional Approach for Iris Recognition,” WSEAS Trans. Inf. Sci. Appl., vol. 11, pp. 61-71, 2014.

[28] J. K. Pillai, V. M. Patel, R. Chellappa, and N. K. Ratha, "Secure and Robust Iris Recognition Using Random Projections and Sparse Representations," IEEE Trans. Pattern Anal. Mach. Intell., vol. 33, no. 9, pp. 1877-1893, Sep. 2011.

[29] N. Salanke, N. Maheswari, and A. Samraj, "An enhanced intrinsic biometric in identifying people by photopleythsmography signal," Proc. Fourth, 2013.

[30] S. Swati and D. Gupta Deepak, "Iris recognition using gabor," Int. J. Comput. Technol. Appl., vol. 4, no. 1, pp. 1-7, 2013.

[31] U. K. Hassan, N. M. Nawi, and S. Kasim, "Classify a protein domain using sigmoid support vector machine," in Information Science and Applications (ICISA), 2014 International Conference, IEEE, May. 2014. pp. 1-4.

[32] U. K. Hassan, N. M. Nawi, and S. Kasim, A. A, Ramli, M. F. M. Fudzee, and M. A. Salamat,"Classify a Protein Domain Using SVM Sigmoid Kernel". In Recent Advances on Soft Computing and Data Mining, pp. 143-151, Springer, Cham.

[33] S., Ismail, R. M., Othman, S., Kasim, R., Hassan, H., Asmuni, and J., Taliba, "Pairwise protein substring alignment with latent semantic analysis and support vector machines to detect remote protein homology", International Journal of Bio-Science and BioTechnology, Volume 3, Issue 3, 2011, Pages 17-34.

[34] F. M., Abdullah, R. M., Othman, S., Kasim, R., Hashim, R., Hassan, H., Asmuni, and J., Taliba, "An Optimal Mesh Algorithm for Remote Protein Homology Detection", Communications in Computer and Information Science, Volume 151 CCIS, Issue PART 2, 2011, Pages 471-497.

[35] S., Ismail, R. M., Othman, S., Kasim, R., Hassan, H., Asmuni, and J., Taliba, "Pairwise protein substring alignment with latent semantic analysis and support vector machines to detect remote protein homology" Communications in Computer and Information Science,Volume 151 CCIS, Issue PART 2, 2011, Pages 526-546.

[36] F. M., Abdullah, R. M., Othman, S., Kasim, R., Hashim, R., Hassan, H., Asmuni, and J., Taliba, "An Optimal Mesh Algorithm for Remote Protein Homology Detection", International Journal of Bio-Science and Bio-Technology, Volume 3, Issue 2, June 2011, Pages 13-38.

[37] S., Kasim, M. F. M., Fudzee, S., Deris, S., and R. M., Othman, "Gene Function Prediction Using Improved Fuzzy c-Means Algorithm", In Information Science and Applications (ICISA), 2014 International Conference on (pp. 1-4). IEEE.

[38] S., Kasim, S., Deris, S., and R. M., Othman, "Multi-stage filtering for improving confidence level and determining dominant clusters in clustering algorithms of gene expression data", Computers in biology and medicine, 43(9), 1120-1133, 2013.

[39] S., Kasim, S., Deris, S., and R. M., Othman, "A new computational framework for gene expression clustering", In International Conference on Advanced Data Mining and Applications (pp. 603610). Springer, Berlin, Heidelberg, 2010. 